\title{
Comparison of different spectral population models
}

\section{Mina Koleva ${ }^{1,2} \dagger$, Philippe Prugniel ${ }^{1,3}$, Pierre Ocvirk $^{4}$, Damien Le Borgne ${ }^{4}$, Igor Chilingarian ${ }^{1,5}$ and Caroline Soubiran ${ }^{6}$}

${ }^{1}$ Université Lyon 1, Observatoire de Lyon, 9 av. Ch. André, St. Genis Laval, F-69230, France; CNRS, UMR 5574

${ }^{2}$ Department of Astronomy, St. Kl. Ohridski University of Sofia, 5 J. Bourchier Blvd., BG-1164 Sofia, Bulgaria

${ }^{3}$ Observatoire de Paris, GEPI, 61 Ave. de l'Observatoire, Paris, 75014, France

${ }^{4}$ CEA Saclay/Service d'Astrophysique Orme des Merisiers, Bat 709, Gif-sur-Yvette Cedex, F-91191, France

${ }^{5}$ Sternberg Astronomical Institute,Moscow University,13 Universitetskij pr., 119992, Moscow, Russia;

${ }^{6}$ Observatoire de Bordeaux,2 rue de l'Observatoire, 33270, Floirac, France

\begin{abstract}
We have compared simple stellar populations (SSPs) generated with different population synthesis tools: BC03, Vazdekis and Pégase.HR and different stellar libraries: ELODIE3.1, SteLib and MILES. We find that BC03/SteLib SSPs are biased toward solar metallicity, however Pégase.HR/ELODIE3.1 and Vazdekis/MILES are extremely consistent. The extensive coverage of the space of atmospheric parameters in the large stellar libraries allows precise synthesis for a large range of ages $(0.1 \ldots 10 \mathrm{Gyr})$ and metallicities $(-2 \ldots+0.4$ dex $)$ limited by the quality of the determination of stellar parameters (temperature scale of the giants).
\end{abstract}

Keywords. galaxies: stellar content, galaxies: evolution, techniques: spectroscopic

\section{Introduction}

There are different methods to derive the parameters of a stellar population (SSPequivalent age and metallicity, or population history) using spectra integrated along the line-of-sight. In this paper we are using full spectrum fitting (i.e. fitting each pixel). We want to estimate the reliability of this approach and to investigate the consistency between the different spectral population models and stellar libraries.

Unlike the spectrophotometric indices, this method uses all the information in the spectrum and is therefore more sensitive (Koleva et al. 2006). Though, as well as indices, our approach is insensitive to the shape of the continuum (plugged with flux calibration errors and extinction uncertainties). The main ingredients of our method are the stellar library, the population model and the minimisation procedure.

We have tested each of these components comparing different stellar libraries (SteLib, Le Borgne 2003; ELODIE3.1, Prugniel \& Soubiran, 2001, 2004; MILES, Sánchez-Blázquez 2006) injected in different population synthesis models (BC03, Bruzual \& Charlot, 2003; Pégase.HR, Le Borgne at al. 2004; Vazdekis 1999) using different minimisation procedures (STECKMAP, Ocvirk et al. and NBursts, Chilingarian et al., this conference). As a reference we have used Pégase.HR evolutionary code with ELODIE3.1 (new version to be released soon). 

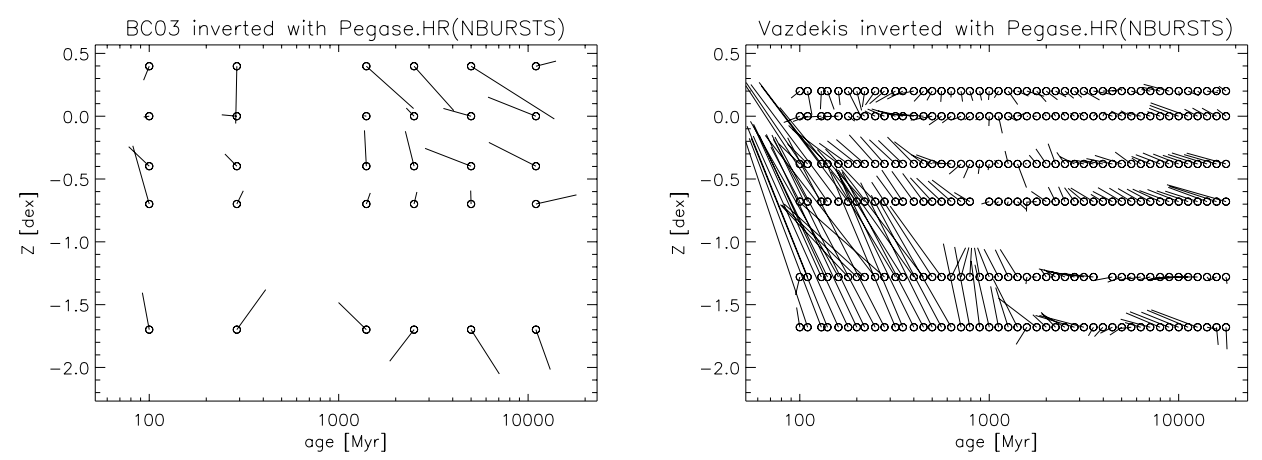

Figure 1. Inversion of (a)Vazdekis-MILES and (b)BC03 models using Pégase.HR/ELODIE3.1 models. The dots at the location in Age-Metallicity of the BC03 templates are connected to the solution found by inversion.

\section{Results}

(1) The insensitivity of the fitting algorithm to the shape of the continuum produce more robust results;

(2) The comparison of BC03 model against Pégase.HR (Fig. 1a) shows the importance of the quality of the libraries (in terms of number of stars and coverage of the parametric space). Owing to the small number of stars in SteLib, BCO3 models of super- and sub-solar metallicities are using almost solar spectra, and the inversion based on more complete libraries (ELODIE3.1 or MILES) converges toward solar;

(3) The Pégase.HR and Vazdekis-MILES models (Fig. 1b) are globally consistent, and a systematic difference between the predicted ages at solar metallicity may be accounted by differences in the evolutionary tracks of temperature calibration of the red giants (a difference of $200 \mathrm{~K}$ explains all the differences);

(4) The difference between Vazdekis-MILES and Pégase.HR-ELODIE3.1 metal poor and young SSPs is related to the lack of hot stars with low metallicity in the stellar libraries. The spectra in this part of the diagram are extrapolated;

(5) The two used inversion programs STECKMAP and NBursts agree well;

Other validation tests using Galactic clusters are presented in the poster by (Koleva et al., this conference).

Acknowledgements. We are grateful to the financial support, provided by the IAU to $\mathrm{MK}$ and IC.

\section{References}

Bruzual, G. \& Charlot, S., 2003, MNRAS 344, 1000

Koleva, M. et al., 2006, astro-ph/0602362

Leborgne, J.-F. et al., 2003, A\&A 402, 433

Le Borgne, D. et al., 2004, A\&A 425, 881

Lejeune, Th., Cuisinier, F., \& Buser, R., 1997, A\&AS 125, 229

Ocvirk, P. et al., 2006, MNRAS 365, 74

Prugniel, Ph. \& Soubiran, C.,2001, A\& A, 369, 1048

Prugniel, Ph. \& Soubiran, C., 2004, astro.ph-/0409214

Sánchez-Blázquez, P. et al., 2006, MNRAS 371, 701

Vazdekis, A., 1999, ApJ 513, 224 